Z. Epileptol. $2022 \cdot 35: 42-45$

https://doi.org/10.1007/s10309-022-00471-2

Angenommen: 18. Januar 2022

Online publiziert: 2. Februar 2022

(c) Der/die Autor(en) 2022

\section{Invasive Ableitung der Insel}

\author{
Christian Dorfer · Johannes Herta · Karl Roessler \\ Universitätsklinik für Neurochirurgie, Medizinische Universität Wien, Wien, Österreich
}

\title{
Zusammenfassung
}

Für die Implantation von Elektroden bei Verdacht auf insuläre Epilepsie stehen verschiedene Zugänge zur Verfügung: 1) offen über eine Kraniotomie, 2) stereotaktisch, wobei die Elektroden orthogonal oder schräg über den Frontallappen und Parietallappen implantiert werden. Die stereotaktische Implantation wird rahmenbasiert, navigationsbasiert oder roboterunterstützt durchgeführt. Die unterschiedlichen Zugänge haben jeweils Vor- und Nachteile in Bezug auf die Anatomie, Funktion und Abdeckung des Inselkortex. Diese Aspekte werden in dieser Übersichtsarbeit ausgeführt.

\section{Schlüsselwörter}

Tiefenelektroden · Insuläre Epilepsie · Invasives Monitoring · SEEG · Stereotaxie

Insuläre Epilepsien sind ein besonders herausforderndes Thema unter den pharmakoresistenten Epilepsien [4]. Ihre besondere anatomische Lage am Grund der Sylvischen Furche bedeckt vom frontalen, temporalen und parietalen Operculum sowie den Ästen der A. cerebri media (ACM) macht den Zugang zur Insel schwierig und morbiditätsbehaftet [18]. Trotzdem können ein gutes Ergebnis und eine hohe Rate an Anfallsfreiheit postoperativ erreicht werden, wobei häufig eine invasive $A b$ leitung der Insel notwendig ist [2, 7-9, $14,18,21]$. Hierfür werden verschiedenste Techniken beschrieben, welche in dieser Übersichtsarbeit beleuchtet werden.

\section{Invasive EEG-Ableitung der Insel}

Die invasive Abklärung mittels Elektroden ist gut etabliert, und die Rolle der Insel bei der Entstehung von Anfällen hat in den letzten Jahren ein zunehmendes Interesse erfahren [10, 15]. Für die Implantation von Elektroden wurden zahlreiche Zugänge beschrieben, von denen jeder Vor- und Nachteile aufweist. Diese Zugänge sind: i) Implantation über eine Kraniotomie unter direkter Sicht [19], stereotaktisch orthogonal $[10,20]$, stereotaktisch von schräg hinten [17] oder von schräg vorne [1] sowie kombiniert von schräg vorn und hinten
$[1,19]$. Bei den stereotaktischen Methoden können dieseZugänge rahmenbasiert, navigationsbasiert und roboterunterstützt erreicht werden [1, 17, 20]. Für jeden dieser Zugänge bleibt das Risiko eines „undersampling" aufgrund der anatomischen Form und Lage der Insel gegeben.

\section{Kraniotomie und Implantation unter direkter Sicht}

Bei dieser Methode werden die Elektroden nach Eröffnung über eine große Kraniotomie und Spalten der Sylvischen Fissur mit mikrochirurgischen Techniken entweder in die Insel mittels Tiefenelektroden und/oder auf die Inseloberfläche mittels Streifenelektroden platziert [12, 22]. Hierbei werden die Tiefenelektroden mithilfe der Navigation implantiert, wobei die Navigation entweder nur eine Orientierungshilfe darstellt und die Elektroden freihändig platziert werden oder ein Navigationsarm als Führung der Elektrode genutzt wird $[13,19]$.

Der Vorteil dieser Methode liegt in der Möglichkeit den perisylvischen Temporalund Frontallappen großzügig mit subduralen Elektroden abdecken zu können. Da diese Kortexareale bei den insulären Epilepsien häufig eine bedeutende Rolle spielen und sich die Möglichkeit eines kortika- 


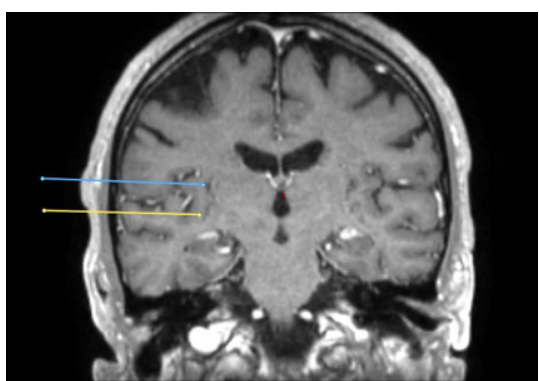

Abb. $1 \Delta$ Dieses MRT (koronar, T1 + KM) zeigt 2 orthogonale Trajekte durch die Sylvische Furche in die Insel

len Mappings erschließt, wird diese Methode deshalb von einigen Zentren unter bestimmten Umständen bevorzugt. Außerdem lässt sich über diesen Zugang sowohl der mediale als auch der laterale Anteil der Insel gut abdecken, was mit den stereotaktischen Methoden aufwendiger ist [16].

Ist eine Abdeckung mittels subduraler Elektroden nicht notwendig, sind stereotaktisch implantierte Tiefenelektroden eine gute Alternative. Die Spaltung der Sylvischen Furche und Manipulation der Äste der A. cerebri media bergen die Gefahr von Ischämien und Kontusion im Temporal-, aber v. a. Frontallappen durch deren Retraktion mit einem Hirnspatel. Außerdem sind die Elektroden, die so implantiert werden, meistens orthogonal implantiert, sodass nur 2 Kontakte tatsächlich den Inselkortex ableiten können [19]. Eine Ausnahme stellt hier die Technik von Weil et al. dar, welche die Implantation über den Apex der Insel entlang der Längsachse vorschlagen [22]. Um die Abdeckung des Inselkortex zu erhöhen, schlagen Park et al. vor, Streifenelektroden auf den Inselkortex nach Spaltung der Sylvischen Furche zu legen [16].

\section{Stereotaktisch orthogonale Implantation von Tiefenelektroden (a Abb. 1)}

Dieser Zugang stellt die klassische Methode zur invasiven Abklärung der Insel dar und basiert in den Grundzügen auf der Methode von Talairach und Bancaud [20]. Die Orientierung der Elektroden ist normal zur Sagittalebene, welche definiert ist durch die Verbindungslinie zwischen vor-
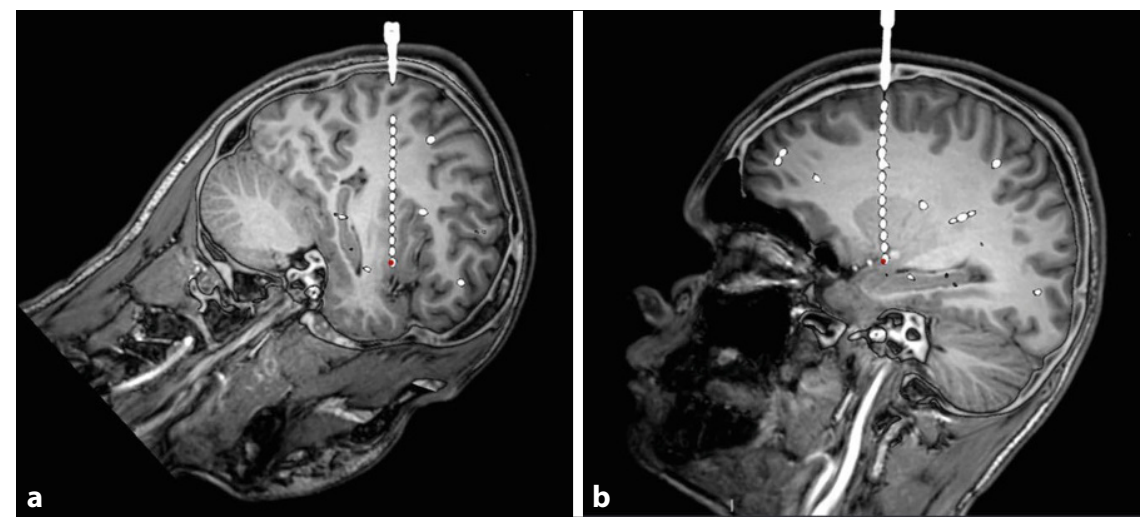

Abb. $2 \Delta$ Dieses MRT (T1 ohne KM) zeigt eine Tiefenelektrode über einen schrägen Zugang von parietal (a) und eine über einen schrägen Zugang von frontal (b) in die Insel

derer und hinterer Kommissur. Der Vorteil dieser Methode liegt in ihrer sehr breiten klinischen Verbreitung und Erfahrung in der Interpretation der Ergebnisse. Die Technik selbst hat sich von der anfänglichen Verwendung des Talairach-Rahmens über andere Rahmensysteme in Kombination mit den jüngst eingeführten Robotern stetig weiterentwickelt [3].

Der Nachteil liegt in der Notwendigkeit, die Sylvische Furche mit all ihren Gefäßen durchkreuzen zu müssen und dabei die gefäßführende Pia mater 2-mal perforieren zu müssen. Diese Methode erfordert daher einen sehr hohen Grad an Genauigkeit. Zwar lässt sich diese Genauigkeit durch eine intraoperative Katheterangiographie oder andere koregistrierte vaskuläre Bildgebung gut erreichen, kann aber auch komplex, zeitaufwendig und für manche Neurochirurgen zu schwerfällig sein [5].

\section{Schräger Zugang von posterior (• Abb. 2a)}

Durch die zunehmende Verwendung von anderen als dem von Talairach und Bancaud entwickelten Stereotaxierahmen (Leksell, CRW) sowie die zunehmende Verbreitung von navigations- bzw. robotergestützten Implantationstechniken haben sich die schrägen Zugänge für die Inselelektroden entwickelt [1, 17]. Diese schrägen Trajekte können entweder von posterior, d. h. transparietal, oder von anterior, d. h. transfrontal, durchgeführt werden.

Die schrägen Zugänge haben gegenüber dem orthogonalen Zugang den Vor- teil, dass man die Sylvische Fissur mit ihren Gefäßen nicht durchkreuzen muss. Grundsätzlich können über einen posterioren Zugang die hinteren Anteile und über einen anterioren Zugang die vorderen Inselanteile besser abgedeckt werden [11]. Durch die spezielle anatomische Form der Insel lassen sich aber über den posterioren Zugang tendenziell größere Anteile als über den anterioren Zugang erreichen. Gegenüber den orthogonalen Trajektorien scheinen beide schrägen Zugänge ein höheres Kontakt/Elektroden-Verhältnis zu erlauben, d. h. die Anzahl der inselrelevanten Kontakte pro implantierter Elektrode ist höher, was weniger Elektroden notwendig macht [11].

Der Nachteil des posterioren Zugangs liegt in manchen Fällen darin, dass die gleichzeitige Platzierung von Elektroden im Temporal- oder Frontallappen lagerungsbedingt in Abhängigkeit von der verwendeten Technik schwieriger sein kann. Temporal- und Frontallappen müssen aber vielfach im Rahmen der invasiven Abklärung abgedeckt werden. Des Weiteren sind die Sulci im Parietallappen meist sehr tief und folgen in ihrer Anordnung keiner einheitlichen Richtung, was die Wahl eines sicheren Trajektes, welches die Sulci nicht kreuzen soll, schwierig gestalten kann.

\section{Schräger Zugang von anterior (- Abb. 2b)}

Der anteriore Zugang durch den Frontallappen wird von manchen Zentren bevorzugt $[1,6,19]$. Er hat den anatomischen Vorteil, dass der Eintrittspunkt frontal meist in einem nicht eloquenten Areal 
liegt und der anatomische Verlauf der Sulci parallel verläuft. Meist sind nur der Verlauf und die Länge des Sulcus frontalis superior zu beachten. Gleichzeitig decken die proximalsten Elektrodenkontakte auf ihrem Weg auch Teile des Frontallappens $a b$, welcher relativ häufig eine Rolle bei Epilepsien der Insel spielt.

Als Nachteil wird von manchen Autoren bei beiden schrägen Zugängen die beschränkte mediolaterale Abdeckung der Insel genannt [6].

\section{Komplikationen}

Auch wenn die Insel aufgrund ihrer besonderen anatomischen Lage eine Herausforderung für die Implantation von Tiefenelektroden darstellt, ist die Komplikationsrate unter Anwendung der geeigneten Techniken und der notwendigen Erfahrung nicht höher als in anderen Lokalisationen [1, 3,5]. Dabei ist es nicht wichtig, welche Technik im Speziellen verwendet wird, sondern dass die Neurochirurginnen und Neurochirurgen die Möglichkeiten und Einschränkungen ihrer jeweils verwendeten Technik genau kennen und die Grenzen der Genauigkeiten bei der Planung der Trajekte antizipieren. So können Tiefenelektroden sowohl über orthogonale [3,5] als auch über schräge Trajektorien [1] sicher in der Insel platziert werden.

\section{Schlussfolgerung}

Die Implantation von Tiefenelektroden in die Insel ist in erfahrenen Zentren sicher möglich. Die Elektroden können entweder über eine Kraniotomie oder stereotaktisch implantiert werden, wobei bei der stereotaktischen Methode verschiedene Richtungen für die Trajekte möglich sind. Diese lassen sich unterscheiden in orthogonale oder schräge Zugänge und werden durch rahmenbasierte, navigationsbasierte oder Robotertechniken unterstützt.

\section{Fazit für die Praxis}

- Die Insel kann über verschiedene Zugänge erreicht werden, wovon jeder seine individuellen Vor- und Nachteile aufweist.

- Der klassische Zugang zur Insel erfolgt über orthogonale Trajekte durch die Sylvische Furche. Die schrägen Trajekte von

\section{Invasive EEG of the insula}

Different approaches for the implantation of electrodes in cases of suspected insular epilepsy are available: 1) implantation under direct vision via a craniotomy with insular exposure and 2) stereotactically, whereby the electrodes are implanted orthogonally or obliquely over the frontal lobes and parietal lobes. The stereotactic implantation of depth electrodes can be performed frame-based, navigation-based or with the help of robotic guidance. The different approaches each have advantages and disadvantages with respect to the anatomy, function and coverage of the insular cortex. These aspects are discussed in this review article.

\section{Keywords}

Depth electrodes · Insular epilepsy · Invasive monitoring · SEEG · Stereotaxy

anterior und posterior meiden ein Durchkreuzen der Sylvischen Furche und haben sich später entwickelt.

- Klassische Stereotaxiemethoden sowie modernste Neuronavigation und Robotik kommen bei der Implantation von Tiefenelektroden zum Einsatz.

\section{Korrespondenzadresse}

Assoc. Prof. PD Dr. Christian Dorfer

Universitätsklinik für Neurochirurgie, Medizinische Universität Wien

Währinger Gürtel 18-20, 1090 Wien, Österreich christian.dorfer@meduniwien.ac.at

Funding. Open access funding provided by Medical University of Vienna.

\section{Einhaltung ethischer Richtlinien}

Interessenkonflikt. C. Dorfer, J. Herta und K. Roessler geben an, dass kein Interessenkonflikt besteht.

Für diesen Beitrag wurden von den Autoren keine Studien an Menschen oder Tieren durchgeführt. Für die aufgeführten Studien gelten die jeweils dort angegebenen ethischen Richtlinien.

Open Access. Dieser Artikel wird unter der Creative Commons Namensnennung 4.0 International Lizenz veröffentlicht, welche die Nutzung, Vervielfältigung, Bearbeitung, Verbreitung und Wiedergabe in jeglichem Medium und Format erlaubt, sofern Sie den/die ursprünglichen Autor(en) und die Quelle ordnungsgemäß nennen, einen Link zur Creative Commons Lizenz beifügen und angeben, ob Änderungen vorgenommen wurden.

Die in diesem Artikel enthaltenen Bilder und sonstiges Drittmaterial unterliegen ebenfalls der genannten Creative Commons Lizenz, sofern sich aus der Abbildungslegende nichts anderes ergibt. Sofern das betreffende Material nicht unter der genannten Creative Commons Lizenz steht und die betreffende Handlung nicht nach gesetzlichen Vorschriften erlaubt ist, ist für die oben aufgeführten Weiterverwendungen des Ma- terials die Einwilligung des jeweiligen Rechteinhabers einzuholen.

Weitere Details zur Lizenz entnehmen Sie bitte der Lizenzinformation auf http://creativecommons.org/ licenses/by/4.0/deed.de.

\section{Literatur}

1. Afif A, Chabardes S, Minotti L, Kahane P, Hoffmann D (2008) Safety and usefulness of insular depth electrodes implanted via an oblique approach in patients with epilepsy. Neurosurgery 62(5):ONS471-ONS480

2. Alexander H, Cobourn K, Fayed I et al (2019) Magnetic resonance-guided laser interstitial thermal therapy for the treatment of nonlesional insular epilepsy in pediatric patients: thermal dynamic and volumetric factors influencing seizure outcomes. Childs Nerv Syst 35:453-461

3. Alomar S, Mullin JP, Smithason S, GonzalezMartinezJ(2018) Indications, technique, and safety profile of insular stereoelectroencephalography electrode implantation in medically intractable epilepsy. J Neurosurg 128:1147-1157

4. Bouthillier A, Nguyen DK (2017) Epilepsy surgeries requiring an opoerculoinsular cortectomy: operative technique and results. Neurosurgery 81:602-612

5. Cardinale F, Cossu M, Castana L (2013) Stereoelectroencephalography: surgical nmethodology, safety, and stereotactic application accuracy in 500 procedures. Neurosurgery 72:353-366

6. Desai A, Bekelis K, Darcey TM, Roberts DW (2012) Surgical techniques for investigating the role of the insula in epilepsy: a review. Neurosurg Focus 32:E6

7. Gireesh ED, Lee K, Skinner H et al (2020) Intracranial EEG and laser interstitial thermal therapy in MRInegative insular and/or cingulate epilepsy: case series. J Neurosurg. https://doi.org/10.3171/2020. 7.JNS201912

8. Guenot M, Isnard J, Catenoix H et al (2011) SEEG-guided RF-thermocoagulation of epileptic foci: a therapeutic alternative for drug-resistant nonoperable partial epilepsies. Adv Tech Stand Neurosurg 36:61-78

9. Irislimane M, Mathieu D, Bouthillier A et al (2013) Gamma knife surgery for refractory insular cortex epilepsy. Stereotact Funct Neurosurg 91:170-176

10. Isnard J, Guenot M, Sindou M, Mauguiere F (2004) Clinical manifestations of insular lobe seizures: a stereo-electroencephalographic study. Epilepsia 45:1079-1090 
11. Machetanz K, Grimm F, Wuttke TV, Kegele J, Lerche $\mathrm{H}$, Tatagiba M, Rona S, Gharabaghi A, Honegger J, Naros G (2021) Frame-based and robotassisted insular stereoelectroencephalography via an anterior or posterior oblique approach. J Neurosurg. https://doi.org/10.3171/2020.10. JNS201843

12. Malak R, Bouthillier A, Carmant L, Cossette $P$, Giard N, Saint-Hilaire JM (2009) Microsurgery of epileptic foci in the insular region. Clinical article. JNeurosurg 110:1153-1163

13. Mehta AD, Labar D, Dean A (2005) Frameless stereotactic placement of depth electrodes in epilepsy surgery. J Neurosurg 102:1040-1045

14. Mullatti N, Landre E, Mellerio C et al (2019) Stereotactic thermocoagulation for insular epilepsy: lessons from successes and failures. Epilepsia 60:1565-1579

15. Nguyen DK, Nguyen DB, Malak R, Leroux JM, Carmant L, Saint-Hilaire JM (2009) Revisiting the role of the insula in re-fractory partial epilepsy. Epilepsia 50:510-520

16. Park YS, Lee YH, Shim KW, Lee YJ, Kim HD, Lee JS (2009) Insular epilepsy surgery under neuronavigation guidance us-ing depth electrode. Childs Nerv Syst 25:591-597

17. Robles SG, Gelisse P, El Fertit H, Tancu C, Duffau H, Crespel A (2009) Parasagittal transinsular electrodes for stereo-EEG in temporal and insular lobe epilepsies. Stereotact Funct Neurosurg 87:368-378

18. Ryvlin P, Nugyen DK (2021) Insular seizures and epilepsies: ictal semiology and minimal invasive surgery. Curr Opin Neurol 34:153-165

19. Surbeck W, Bouthillier A, Weil AG, Crevier L, Carmant L, Lortie A (2011) The combination of subdural and depth electrodes for intracranial EEG investigation of suspected insular (perisylvian) epilepsy. Epilepsia 52:458-466

20. Talairach J, Bancaud J (1973) Stereotactic approach to epilepsy. Methodology of anatomo-functional stereotaxic investigations. Prog Neurol Surg 5:297-354

21. von Lehe M, Parpaley Y (2017) Insular cortex surgery for the treatment of refractory epilepsy. JClin Neurophysiol 34:333-339

22. Weil A, Fallah A, Lewis EC, Bhatia S (2016) Medically resistant pediatric insular-opercular/perisylvian epilepsy. Part 1: invasive monitoring using the parasagittal transinsular apex depth electrode. JNeurosurg Pediatr 18:511-522

\section{Hermann-Thomas Steffen}

\section{Pflege in der Epileptologie}

Wissen, Versorgung, Praxiskompetenz (Neurologische Pflege)

\section{Stuttgart: W. Kohlhammer 2021, 225 S., (ISBN: 978-3-17-037302-0), Gebunden 24,95 EUR}

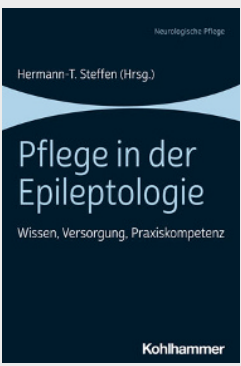

Ein aktuelles Buch über die Besonderheiten der Pflege von Menschen mit Epilepsie fehlte im deutschsprachigen Raum seit vielen Jahren. Das erste vergleichbare Werk ist Ende des 19. Jahrhunderts erschienen (Wildermuth H. Über die Aufgaben des Pflegepersonals bei Epileptischen. Halle a. S., C. Marhold 1897), das letzte vor über 70 Jahren (Schlaich L. Die Pflege und Erziehung Geistesschwacher und Epileptischer in Anstalten der Inneren Mission. Entwurf eines Lehrbuches für Pfleger, Erzieher und Lehrmeister. Bielefeld, L. Bechauf 1953).

Der Herausgeber, Hermann-Thomas Steffen (* 1962), ist examinierter Krankenpfleger und war bis 2011 in verschiedenen klinischen Bereichen (Epilepsie und Mehrfacherkrankungen, Epilepsiechirurgie) am EpilepsieZentrum Bethel tätig. Danach war er ebenfalls in Bethel in der Pflege- und Versorgungsforschung aktiv und seit 2014 Lehrkraft an der Fachhochschule der Diakonie in Bielefeld. Seit 2017 ist er Professor für Gesundheitswissenschaften und Versorgungsforschung an der Fachhochschule der Diakonie. Er wird unterstützt von 10 Koautorinnen und Koautoren, bis auf eine Ausnahme alle aus BethelBielefeld.

Das Buch ist in zehn Kapitel unterteilt. Auf einen kurzen Abriss der medizinischen Grundlagen folgen „Anfälle beobachten und erste Hilfe bei Anfallsereignissen“, „Pflege in der Kinderepileptologie“, „Pflege in der Behandlung von jungen Erwachsenen mit Epilepsie“, „Pflege bei Menschen mit Epilepsie im Erwachsenenalter", „Pflege von Erwachsenen mit einer komplexen Behinderung und Epilepsie“, „Pflege in der psychosomatischen Epileptologie“, „Pflege in der Epilepsiechirurgie", "Pflege in der epileptologischen Rehabilitation" und „Epilepsieberatung in der
Pflege". In allen Kapiteln werden die neuen Anfalls- und Epilepsie-Klassifikationen der ILAE von 2017 sowie die aktuelle Literatur berücksichtigt.

Die Stärke des Buches liegt sicherlich in der Tatsache, dass es aus einem der führenden deutschsprachigen Epilepsiezentren kommt, in dem Mitarbeiterschulung seit Jahrzehnten ein Schwerpunkt war und ist. Kleinigkeiten lassen sich in jedem Werk finden und bei einer nächsten Auflage gegebenenfalls korrigieren. So sollte Kaliumbromid in der ansonsten strikt alphabetisch gegliederten Tabelle 1.1 der Antiepileptika nicht an erster Stelle genannt werden, und die seit $2021 \mathrm{zu}$ gelassenen Wirkstoffe Cenobamat und Fenfluramin fehlen. Warum es am Ende jeden Kapitels neudeutsch "Lessons learned" anstelle z.B. "Das Wichtigste in Kürze" oder schlicht "Zusammenfassung" heißt, erschließt sich möglicherweise nicht nur mir nicht. Den Gender-Stern finde ich immer noch nervig, der blass-graue Druck der digitalen Literaturquellen (in den Vorlagen hellblau) erleichtert das Lesen nicht und ich würde mich über mehr Abbildungen freuen. Unklar ist auch, warum im Impressum des Buches 2021 bei einem tatsächlichen Erscheinungsdatum im Januar 2022 angegeben ist.

Insgesamt jedoch ein gelungenes Buch für alle an der Pflege von Menschen mit Epilepsie beteiligten Fachpersonen. Es ist sicher als Baustein einer weiteren Professionalisierung der Pflege geeignet, wobei mir als Fernziel vorschwebt, was ich z.B. in den USA erlebt habe, wo bei der gemeinsamen Visite besprochen wurde, dass ein Patient auf ein bestimmtes Medikament eingestellt wird und der Rest eigenständig und eigenverantwortlich durch die Pflege erfolgte.

Günter Krämer, Zürich 